\section{PEDIATRIC HOSPITALIZATIONS ASSOCIATED WITH 2009 PANDEMIC INFLUENZA A (H1N1) PNEUMONIA IN A SECONDARY CARE HOSPITAL}

\author{
S. Águeda, Â. Machado, C. Monteiro, J. Cunha, \\ I. Quintal \\ Department of Pediatrics, Centro Hospitalar \\ do Tâmega e Sousa - Unidade Hospital Padre \\ Américo, Penafiel, Portugal
}

Background and aims: In 2009 an outbreak of a respiratory illness later proved to be caused by novel influenza A virus (H1N1) was identified all around the world. Our goal was to evaluate children with pneumonia in whom $\mathrm{H} 1 \mathrm{~N} 1$ infection was diagnosed by reverse-transcriptase reaction assay, to determine its frequency and to evaluate the clinical presentation, chest x-ray patterns, laboratorial results, treatment, underlying conditions and outcome.

Methods: We conducted a retrospective study involving children who were hospitalized between September and December 2009 at our secondary care Hospital.

Results: Forty children were hospitalized with pneumonia, 13 of them with $\mathrm{H} 1 \mathrm{~N} 1$ infection $(32,5 \%)$. The mean age was 3,42 $\pm 3,77$ years and $61,5 \%$ were male. The main complains at admission was fever and cough (92,3\%), rhinorrhea $(61,5 \%)$, respiratory distress $(46,2 \%)$ and feeding difficulty $(30,8 \%)$. Oseltamivir was used in all patients, alone $(30,8 \%)$ or associated with an antibiotic $(69,2 \%)$. In $46,2 \%$ of patients was initiated in less than 48 hours. The mean length of hospitalization was $5,54 \pm 1,39$ days. There were bilateral infiltrates on chest $x$-ray in $38,5 \%$. Laboratorial results showed leukocytosis in $15,4 \%$ but the majority $(53,8 \%)$ did not have any analytic change. There was a history of asthma in $15,4 \%$ and of immunosuppression plus chronic pulmonary disease in $23,1 \%$. There were no records of morbidity or mortality.

Conclusions: The outcome was favorable in all admitted patients. This study will be continued within the next months to have a more accurate view of the epidemiology and outcome of the H1N1 infection in children with pneumonia.

\section{POSITIVE TUBERCULIN SKIN TEST (TST) IN CHILDREN IN HERAKLION, CRETE, GREECE: RESEARCH AND CONFRONTATION}

M.G. Avanidi, A. Christoforaki, A. Kleisarchaki, V. Aggelakou, G. Vlachaki, E. Kokkori

Pediatrics, General Hospital of Venizelion, Heraklion, Greece

Background and aims: There is a remarked dramatic increase of the incidence of tuberculosis in childhood the recent twenty years, after the continually decreased the last decades. This fact provokes the sensitization of health services. TST is one of the most useful diagnostic methods of restriction individuals infected of mycobacterium tuberculosis. The goal of this study was to record the laboratory tests and the treatment in children with positive TST in Heraklion region.

Methods: Retrospective analysis of children younger than 14 years old with positive TST, from January 2008 since December 2009 and their follow up.

Results: 25 children aged from 14 months up to 13 years old were hospitalized at this time period due to positive TST. The TST was carried out in $24 \%$ of these children before MMR vaccination, in $40 \%$ before registration at school and in $28 \%$ while investigating adults of their family with positive TST or active tuberculosis. $30 \%$ of them had a positive interferon- $\gamma$ release assays test and $20 \%$ had pathological findings at their X-Ray. $85 \%$ were confronted as latent tuberculosis and there was presented no side effect from the treatment.

Conclusions: The TST in childhood is crucial in early identification and confrontation (management) of the affected people and the restriction of the spread of the tuberculosis infection. 